\title{
Comparison of interventricular septal motion studied by ventriculography and echocardiography in patients with atrial septal defect ${ }^{1}$
}

\author{
THOMAS M. MUELLER, RICHARD E. KERBER, AND MELVIN L. MARCUS \\ From the Cardiovascular Center and the Department of Internal Medicine, University of Iowa College of \\ Medicine and the Iowa City Veterans Administration Hospital, Iowa City, Iowa, USA
}

SUMMARY Abnormal systolic interventricular septal motion is an echocardiographic manifestation of right ventricular volume overload. In order to determine the anatomical basis for this echocardiographic finding, septal motion recorded by left lateral or left anterior oblique ventriculograms was compared with echocardiographic ventricular septal motion. Thirteen patients with secundum atrial septal defects and 7 control patients with trivial or no heart disease were included in the study. We found that on ventriculograms the systolic motion of the interventricular septum was similar in both atrial septal defect and control patients. That is, the cephalic third of the septum moved anteriorly in systole in 9 of the 13 patients with atrial septal defect and in 3 of the 7 controls. The caudal two-thirds of the septum moved posteriorly in all patients. These results are compatible with the theory that argues that the echocardiographic abnormalities of septal motion in patients with atrial septal defect result from an end-diastolic septal flattening or bowing of the septum into the cavity of the left ventricle.

Abnormal systolic interventricular septal motion is a cardinal echocardiographic sign in patients with intra-atrial septal defects (Popp et al., 1969; Diamond et al., 1971). Clinical and experimental studies have suggested that right ventricular volume overload is the cause of abnormal interventricular septal motion (Kerber et al., 1973). There are 3 major hypotheses that attempt to explain the echocardiographic abnormalities of interventricular septal motion in right ventricular volume overload. The first is that with regard to a fixed reference point (location of the echocardiographic transducer on the chest wall), the net motion of the septum will be anterior in systole if there is an anterior motion of the entire heart caused by the ejection of a large right ventricular volume (Meyer et al., 1972; Pearlman et al., 1976). The second hypothesis suggests that in patients with right ventricular volume overload there is caudal displacement of the normal septal 'hinge point' (the point about which the septum pivots, as ventricular ejection occurs), so that a much larger than usual portion of the septum moves anteriorly (Hagan et al., 1974).

1Supported by a Program Project Grant.

Received for publication 18 October 1977
The third hypothesis holds that the interventricular septum may bow into the cavity of the left ventricle at end-diastole to accommodate the right ventricular volume overload. Starting in early systole, when the ventricle begins to assume a more rounded configuration, the septum may appear to move anteriorly (Weyman et al., 1976).

The purpose of this study was to use a nonultrasonic technique, ventriculography, to evaluate the motion of the interventricular septum in patients with intra-atrial septal defects, and to correlate the results with standard $M$ mode echocardiographic examination, in order to assess critically the hypotheses listed above.

\section{Methods}

\section{PATIENT SELECTION}

The records of all patients undergoing catheterisation at Iowa University Hospitals with a diagnosis of intra-atrial septal defect during the period 1 March 1974 to 1 November 1976 were reviewed. All patients who met the following criteria were included in this study: (1) Ostium secundum intraatrial septal defect; (2) availability of cardiac catheterisation data including a left ventriculogram 
taken in the left anterior oblique or left lateral projection; (3) availability of a good quality echocardiographic recording of interventricular septal motion at the level of the mitral valve chordae; (4) lack of significant pulmonary hypertension (pulmonary artery systolic pressure less than $35 \mathrm{mmHg}$ ); (5) lack of other significant or complicating haemodynamic abnormalities, for example mitral regurgitation (Popp et al., 1969; Diamond et al., 1971). All the patients had incomplete right bundlebranch block', but none had other conduction defects such as left bundle-branch block or preexcitation syndromes. None of the patients had previous cardiac surgery or coronary artery disease. Thirteen patients with intra-atrial septal defects met these criteria. The patients who were used as controls were selected on the following basis: (1) discharge diagnosis of no heart disease or trivial haemodynamic defect; (2) availability of cardiac catheterisation data including a left ventriculogram taken in the left anterior oblique or left lateral projection; (3) availability of a good quality echocardiographic recording of interventricular septal motion at the chordal level.

\section{VENTRICULOGRAMS}

The left ventricular cineangiograms were performed in the 45 to $60^{\circ}$ left anterior oblique or the left lateral projection at 60 frames per second. The ventriculograms of 8 patients with intra-atrial septal defect were obtained in the left anterior oblique projection and 5 were obtained in the left lateral projection. Of the control patients, 4 ventriculograms were obtained in the left anterior oblique and 3 in the left lateral projection. The ventriculograms were projected and the ventricular silhouettes drawn superimposed at end-systole and end-diastole. The vertebral bodies were visible on all the cineangiograms and therefore were used as fixed reference points; thus, anterior and posterior interventricular septal motion refer to motion away from or toward the vertebral bodies, respectively. The interventricular septum was divided into thirds by drawing a line from the junction of the aortic valve ring and the interventricular septum to the most caudal point on the diastolic ventricular silhouette. This line was then divided into thirds. We defined the ventriculographic hinge point as that point at which the end-systolic silhouette of the interventricular septum intersected with the enddiastolic silhouette.

Ventricular silhouettes were drawn of one of the first three beats after injection of contrast. No important change in ventricular volume or function would have occurred (Hammermeister and Warbasse, 1973).

\section{ECHOCARDIOGRAMS}

Echocardiographic examinations were performed using a Smith Kline Ekoline 20A ultrasonoscope interfaced with a Honeywell strip chart recorder. A $2.25 \mathrm{MHz}$ focused transducer was used for adults and a 3.75 $\mathrm{MHz}$ transducer was used for smaller children. Echocardiograms were recorded with standard techniques, that is the transducer was placed parasternally and perpendicular to the chest wall in the third or fourth left intercostal space. The patient was supine or in the left lateral decubitus position. In all the patients and controls the mitral valve was identified; then the transducer was directed inferiorly to the level of the chordae tendineae. At this level the determination of systolic interventricular septal motion was made.

Interventricular septal motion was considered to be normal on echocardiography if the left side of the interventricular septum moved posteriorly, that is toward the left ventricular posterior wall during systolic ejection, and abnormal if it moved anteriorly during systolic ejection (type A), or had no anterior-posterior movement during ejection (type B) (Diamond et al., 1971). In most patients an echocardiographic 'sweep' from the chordal level to the aortic root was performed and we determined whether an echocardiographic hinge point was present. We defined this hinge point as that point about which the septum appeared to rotate with the superior portion moving anteriorly in systole and the inferior portion moving posteriorly with systole, as shown in Fig. 1. This determination was made in 10 of the patients with intra-atrial septal defects and in 4 of the controls. The remaining 6 echocardiograms (3 patients and 3 controls) did not contain a technically adequate recording of the sweep from the base to the apex of the ventricle. That is, though the interventricular septal motion was recorded well at the chordal level, it was not recorded sufficiently far superiorly or inferiorly to that point so that a directional difference in the systolic interventricular septal motion at different levels along the septum might be identified.

\section{Results}

\section{PATIENTS SELECTED}

The characteristics of the patients in this study are listed in the Table. The age range of the patients with intra-atrial septal defects was from 1 year to 35 years; 11 of these patients were female. The ratio of pulmonary to systemic blood flow ( $\dot{\mathrm{Q} p} / \dot{\mathrm{Q}} \mathrm{s}$ ) ranged from $2 \cdot 2: 1$ to $4 \cdot 4: 1$. The patients who served as controls ranged in age from 6 to 35 years, and 5 of these 7 were male. Four of the control patients had small interventricular septal defects 
with left to right shunts having a $\dot{\mathrm{Q} p} / \dot{\mathrm{Q}} \mathrm{s}$ ratio of 1.5:1 or less. One patient had minimal pulmonary stenosis with a gradient of $12 \mathrm{mmHg}$ at rest. One patient had a very mild mitral prolapse with a trivial amount of mitral regurgitation and one patient had no evident cardiac disease.

\section{ECHOCARDIOGRAMS}

All of the control patients had normal interventricular septal systolic motion. A hinge point was seen in all 4 of the echocardiograms that were technically adequate to analyse for this feature (Fig. 1). In all 4 the hinge point was observed above the level at

Table

\begin{tabular}{|c|c|c|c|c|c|c|}
\hline Patient & Age & $\operatorname{Sex}$ & $\dot{Q} p / \dot{Q}_{s}$ & Echot & $\begin{array}{l}\text { Hinge point } \\
\text { Echo }\end{array}$ & $\begin{array}{l}\text { Hinge point } \\
\text { Ventriculogram }\end{array}$ \\
\hline \multicolumn{7}{|c|}{$\begin{array}{l}\text { Intra-atrial septal defect } \\
\text { Studied in left anterior oblique }\end{array}$} \\
\hline 1 & 20 & F & $3: 1$ & B & No & No \\
\hline 2 & 35 & $\mathbf{F}$ & $3: 1$ & B & Yes & No \\
\hline 3 & 22 & F & $3 \cdot 4: 1$ & $\mathbf{A}$ & No & Yes \\
\hline 4 & 26 & $\mathbf{F}$ & $2 \cdot 6: 1$ & $\mathbf{A}$ & No & Yes \\
\hline 5 & 33 & F & $4: 1$ & $\mathbf{N}$ & - & Yes \\
\hline 6 & 29 & $\mathbf{M}$ & $3 \cdot 7: 1$ & A & No & Yes \\
\hline 7 & 25 & $\mathbf{M}$ & $2 \cdot 6: 1$ & A & No & Yes \\
\hline & & $\mathbf{F}$ & $4 \cdot 2: 1$ & A & No & Yes \\
\hline \multicolumn{7}{|c|}{ Studied in left lateral } \\
\hline 9 & $1 \mathrm{yr}$ & F & $2 \cdot 9: 1$ & $\mathbf{N}$ & - & No \\
\hline 10 & & $\mathbf{F}$ & $2 \cdot 9: 1$ & $\mathbf{B}$ & - & Yes \\
\hline 11 & & $\mathbf{F}$ & $2 \cdot 7: 1$ & $\mathbf{A}$ & No & Yes \\
\hline 12 & & $\mathbf{F}$ & $2 \cdot 2: 1$ & A & No & No \\
\hline 13 & & $\mathbf{F}$ & $4 \cdot 4: 1$ & $\mathbf{A}$ & No & Yes \\
\hline \multicolumn{7}{|c|}{ Control patients } \\
\hline 14 & 32 & $F$ & Small VSD Qp/Qs $1.4: 1$ & $\mathbf{N}$ & - & Yes \\
\hline 15 & 35 & $\mathbf{M}$ & Small VSD Qp/Qs $1 \cdot 2: 1$ & $\mathbf{N}$ & Yes & No \\
\hline 16 & 18 & $\mathbf{M}$ & Small VSD Qp/Qs $1 \cdot 5: 1$ & $\mathbf{N}$ & Yes & Yes \\
\hline 17 & 26 & $\mathbf{F}$ & Small VSD Qp/Qs $1 \cdot 2: 1$ & $\mathbf{N}$ & Yes & No \\
\hline \multicolumn{7}{|c|}{ Studied in left lateral } \\
\hline 18 & 6 & $\mathbf{M}$ & Minimal PS gradient $12 \mathrm{mmHg}$ & $\mathbf{N}$ & - & Yes \\
\hline 19 & 6 & $\mathbf{M}$ & Normal heart & $\mathbf{N}$ & Yes & No \\
\hline 20 & 17 & $\mathbf{M}$ & Mitral prolapse, mild mitral regurgitation & $\mathbf{N}$ & Yes & No \\
\hline \multicolumn{7}{|c|}{$\begin{array}{l}\text { ^Pulmonary flow (Qp) } \\
\text { Systemic flow (Qs) } \\
\text { †A, anterior systolic septal motion; B, lack of systolic septal motion; N, normal posterior systolic septal motion. } \\
\text { VSD, interventricular septal motion; PS, pulmonary stenosis. }\end{array}$} \\
\hline
\end{tabular}




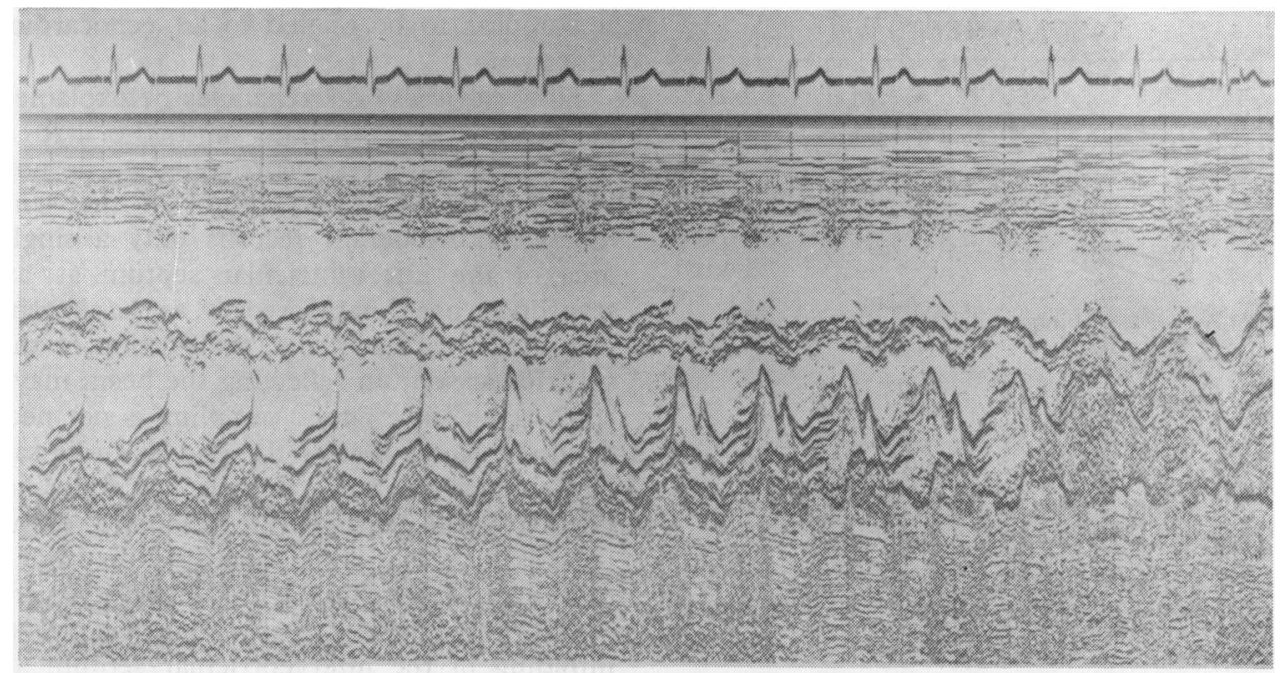

Fig. 2 Patient with intra-atrial septal defect. An echocardiographic sweep from the chordae to the aorta shows that the entire septum moves anteriorly in systole. No hinge point is seen.

which the chordae tendineae were recorded. None of the control patients had any abnormality on their echocardiograms except for 1 patient, case 20 , who had very slight systolic posterior bowing of the mitral valve leaflets suggesting mitral valve prolapse.

Of the 13 patients with intra-atrial septal defects, 11 had abnormal systolic interventricular septal motion. Eight had type A motion (Diamond et al., 1971); that is the left ventricular side of the interventricular septum moved anteriorly or toward the chest wall during systole. Three patients had no net systolic interventricular septal motion and were classified as type B. Two patients had normal systolic interventricular septal motion, a finding occasionally encountered in patients with intraatrial septal defect, and apparently not closely correlated with the volume of the shunt (McCann et al., 1972; Kerber et al., 1973). A hinge point could be identified in only 1 of the 10 patients with intra-atrial septal defect who had a technically adequate echocardiogram for this determination. In this patient, who had type B septal motion, the echocardiographic hinge point appeared to be below the level of the chordae tendineae. In the remaining 9 patients with intra-atrial septal defect, the entire visualised septum from the chordae to the aortic root appeared to move anteriorly by echocardiography (Fig. 2).

\section{VENTRICULOGRAMS}

In all of the controls and the patients with intraatrial septal defect, the ventriculographic motion of the caudal two-thirds of the interventricular septum was always posterior in systole, whether the ventricle was viewed from the left lateral or the left anterior oblique projection. In 9 of the 13 patients with intra-atrial septal defect and 3 of the 7 control patients a ventriculographic hinge point was observed. In the remaining ventriculograms no hinge point was seen. Examples are seen in Fig. 3. Cephalic to the hinge point, the interventricular septum moved anteriorly in systole. However, the level of this ventriculographic hinge point was at or superior to the junction of the cephalic and middle thirds of the septum in all ventriculograms in which it was observed, and no difference in interventricular septal motion was noted between the control patients and the patients with intra-atrial septal defect. Furthermore, the presence of an angiographic hinge point did not correlate with the presence of an echocardiographic hinge point or with the presence of abnormal septal motion at or below the level of the mitral valve chordae seen on the echocardiograms (Table).

\section{Discussion}

The major findings in this study are: (1) there is no evident difference in systolic interventricular septal motion seen in left anterior oblique or left lateral ventriculograms between the patients with intraatrial septal defect and the control subjects; (2) angiographically, the caudal two-thirds of the interventricular septum moved posteriorly during systole in both patients with intra-atrial septal defect and in control subjects, even when there was obvious anterior systolic movement of the inter- 
IASD PATIENTS

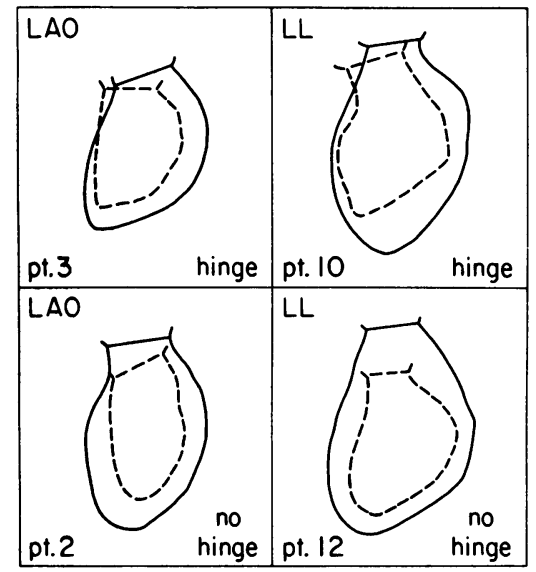

CONTROLS

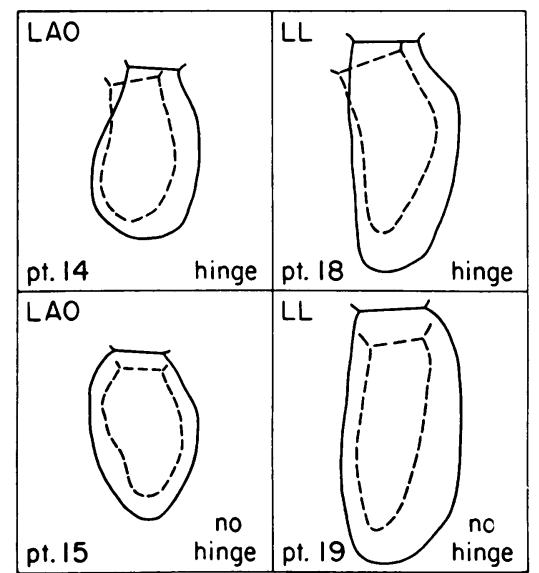

Fig. 3 This figure shows representative end-diastolic (solid line) and end-systolic (broken lines) silhouettes from ventriculograms of patients with intra-atrial septal defect and controls. Examples from the left anterior oblique $(L A O)$ and the left lateral $(L L)$ projections are shown. $A$ hinge point is present in some patients with atrial septal defect and in some controls, and absent in others. The inferior two-thirds of the septum moves posteriorly in systole in all examples, whether or not a hinge point is present.

ventricular septum seen below the mitral valve level on echocardiograms; (3) a hinge point was seen angiographically in 9 of 13 patients with atrial septal defect and in 3 of 7 control subjects, and in none of these was it located below the cephalic third of the septum, even in those patients who had obvious echocardiographic anterior systolic interventricular septal motion well below the chordal level. Thus, the presence of an angiographic hinge point did not appear to be related to an echocardiographic hinge point.

How can these discrepancies be explained? The standard echocardiogram and the left anterior oblique or left lateral ventriculogram do not record interventricular septal motion in the same way. The echocardiogram records only a single small of area of the interventricular septum at any one transducer position. Moreover, as the septum moves during the cardiac cycle, the portion of the interventricular septum reflecting the beam may change so that the end-systolic sampling is not necessarily from the same area as the end-diastolic sampling. The ventriculogram, though recording a larger portion of the septum, is a silhouette technique. It records in profile and, therefore, may not discern movements of small portions of the interventricular septum, especially if there is an irregular shape or infolding of the interventricular septum along its long axis. The relation of the views of the septum by echocardiogram and by left anterior oblique and left lateral ventriculograms can be seen in Fig. 4. The left anterior oblique ventriculogram appears to profile the interventricular septum more accurately than the left lateral. The lateral view was included in our study because, in state of right ventricular volume overload, the enlarged right ventricle may displace the heart such that the interventricular septum is oriented more nearly parallel to the frontal

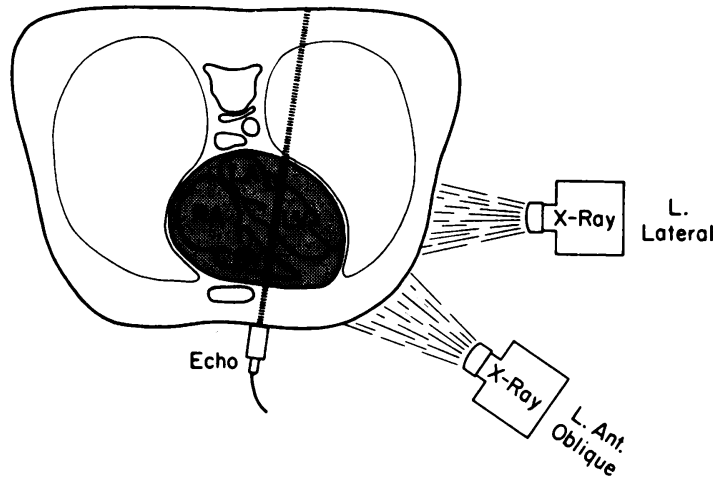

Fig. 4 For comparison, the angles of view of the echocardiographic transducer and the left lateral and left anterior oblique ventriculograms are drawn. The echocardiogram records only a small portion of the ventricular septum, and the area from which the beam reflects may change during cardiac contraction because of motion and rotation of the heart in systole. Ventriculography visualises the septum by a silhouette technique. The left anterior oblique projection profiles the ventricular septum best in a normal heart. However, there may be rotation of the heart in right ventricular volume overload so that the septum becomes more nearly parallel to the frontal plane and is best profiled in the left lateral projection. 
plane (Meyer et al., 1972). It can be seen from examining Fig. 4 that, in either angiographic view, if the interventricular septum moves towards the vertebral bodies the septal movement is posterior in the saggital plane, though the vector of interventricular septal motion may be directed slightly to the right or left.

Three hypotheses have been advanced to explain the echocardiographic findings of abnormal anterior systolic interventricular septal motion in patients with intra-atrial septal defect. The first of these explains abnormal motion by attributing it to an artefact which occurs because the right ventricle is considerably enlarged and displaces the left ventricle posteriorly. During cardiac contraction the entire heart moves anteriorly because of the ejection of a large right ventricular volume (Fig. 5). Therefore, even though the left ventricular side of the interventricular septum is actually contracting normally and moving toward the free posterior

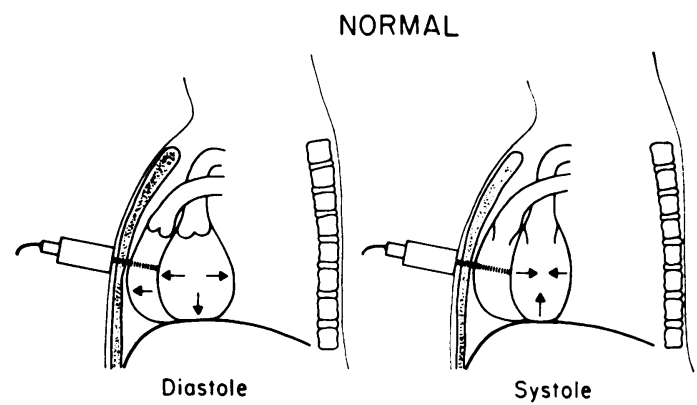

RV VOLUME OVERLOAD

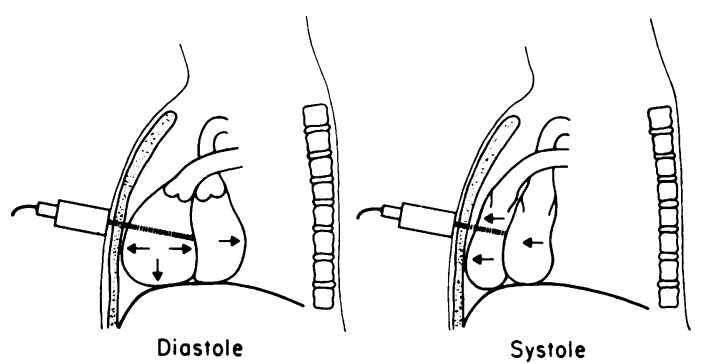

Fig. 5 This figure illustrates the hypothesis that paradoxical septal motion occurs because the entire heart is displaced posteriorly in diastole in right ventricular volume overload (lower left panel), and with ejection of the large right ventricular volume the entire heart swings anteriorly, producing anterior motion with respect to the echocardiographic transducer (lower right). The left ventricle would contract concentrically, and if this theory were correct there would be exaggerated anterior systolic motion of the posterior left ventricular wall. The normal situation is illustrated at the top for comparison. wall of the ventricle, with respect to the transducer, the net motion is anterior. This theory has been proposed by Meyer et al. (1972) and appears to be supported by the more recent work of Pearlman et al. (1976). The theory presupposes concentric left ventricular contraction and would also require very exaggerated anterior motion of the left ventrivular posterior wall during systole, which would occur if the entire heart's anterior motion were sufficient to cause the net interventricular septal motion to be anterior. However, we did not find anterior systolic motion of the interventricular septum angiographically but we did find net posterior systolic movement of at least the lower two-thirds of the left side of the interventricular septum with respect to a fixed radiological reference point in all of our patients with intra-atrial septal defects, as well as in all the control subjects. Moreover, there did not appear to be exaggeration of the posterior wall motion on ventriculograms of the patients with intra-atrial septal defects compared with the controls (Fig. 2).

The second hypothesis stems from the work of Hagan and colleagues (1974) who used a multielement ultrasonic transducer to obtain a long axis cross-sectional cardiac image. They described an echocardiographic hinge point in the interventricular septum. Interventricular septal motion by this technique appeared to be anterior in the cephalic third of the septum and posterior in the middle and caudal thirds in normal subjects. In patients with right ventricular volume overload, the point at which the septum appeared to pivot occurred below the junction of the cephalic and middle thirds of the interventricular septum. Presumably the motion of the entire heart is altered in a similar fashion as shown in Fig. 6. A hinge point was inconstantly present on the ventriculograms which we reviewed, and, even when present, never occurred below the junction of the cephalic and middle thirds of the interventricular septum. Another difference between our angiographic findings and the data obtained with the multielement transducer is that 8 of the 21 patients with right ventricular volume overload examined with the multi-element ultrasound technique seemed to have anterior systolic motion of the entire portion of the interventricular septum that could be recorded with this transducer. However, on ventriculography, we invariably found posterior systolic motion of the inferior two-thirds of the interventricular septum. Movement of the heart about a hinge point on its long axis could account for the anterior motion of the cephalic third of the interventricular septum seen angiographically in some of our patients (Fig. 3). Moreover, the hinge point 


\section{NORMAL Patient}

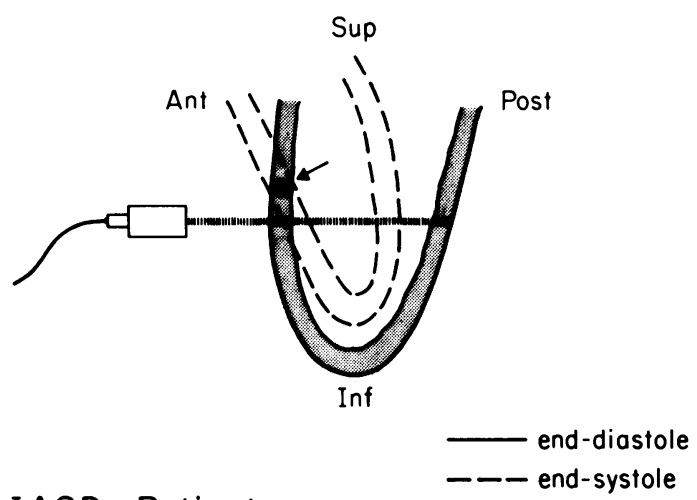

\section{IASD Patient}

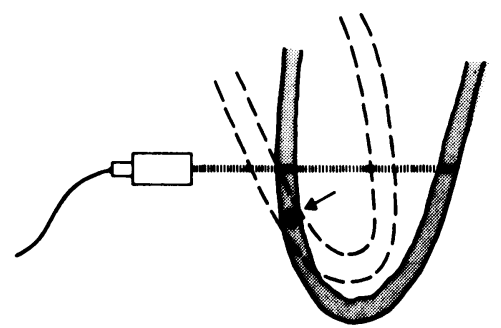

Fig. 6 The hypothesis explaining anterior systolic septal motion by an inferior displacement of a hinge point (marked with a dot and arrow) is illustrated in the figure. The septum appears to move posteriorly in systole in normal patients (upper panel), but anteriorly in right ventricular volume overload (lower panel), because the hinge point has been displaced inferiorly. IASD = intra-atrial septal defect.

hypothesis may be correct if it is combined with another hypothesis, that of an irregular shape of the septum in atrial septal defect patients (see below).

The third hypothesis, proposed by Weyman et al. (1976), is based on evidence gathered from analysis of minor axis cross-section echocardiograms obtained with a mechanical sector-scanner. Weyman and his co-workers showed that in right ventricular volume overload the interventricular septum flattens or bows inward into the cavity of the left ventricle at end-diastole. During the early part of systole, the minor axis cross-section begins to reassume a rounded configuration. Later in systole a relatively concentric contraction ensues. This pattern of contraction is shown diagrammatically in Fig. 7. Our findings are compatible with this mechanism in that invagination of the septum into the left ventricular cavity might not appear on a left lateral or left anterior oblique ventriculogram as abnormal septal motion. As can be seen in Fig. 7, interventricular septal flattening or invagination into
IASD
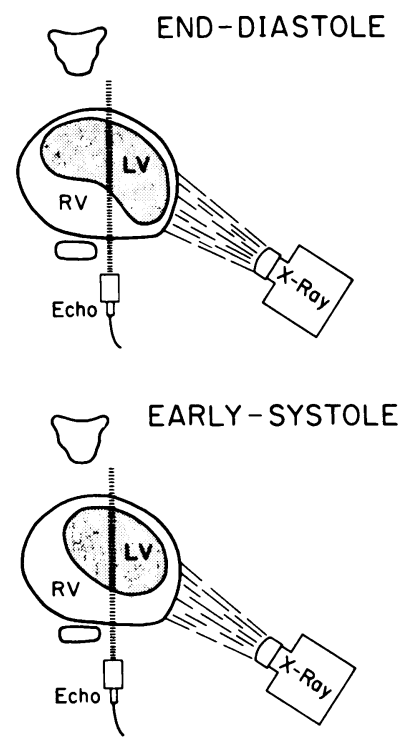

Fig. 7 This figure shows a possible explanation for the discrepancies between echocardiographic and ventriculographic demonstration of septal motion in right ventricular volume overload. End-diastolic bowing of the septum into the cavity of the left ventricle may occur in right ventricular volume overload as shown in a transverse view in the upper panel. In the lower panel the ventricle has begun to assume a rounded configuration in early systole. This alteration in ventricular shape may not be recorded in the silhouette of the ventriculogram because contrast medium in the far right and left anterior portions of the ventricular cavity may overlie and conceal the diastolic septal invagination with respect to the $\mathrm{x}$-ray beam. Thus, the septum may appear to move anteriorly in systole with respect to the ultrasound beam, but posteriorly in systole with respect to the silhouette visualised radiographically.

the cavity of the left ventricle might be obscured by angiographic contrast medium in the anterior 0 portion of the left ventricle overlying the area of invagination with respect to the $x$-ray beam, thereby $\frac{D}{2}$ forming a silhouette that would not show this localised deformity. Moreover, the finding of end- $N$ diastolic septal flattening or bowing in the ventri- $N$ cular minor axis is compatible with the finding of a N septal hinge point in the long axis. That is, the portion of the septum which bows inward at enddiastole appears to move anteriorly in early systole as the ventricle assumes a rounded configuration. $\stackrel{\mathbb{\infty}}{+}$ The lowest point of the invagination appears as an inferiorly displaced hinge point. However, this rationale does not explain the uniform finding by $\vec{\odot}$ Hagan et al. of a hinge point in the cephalic portion $\frac{}{\mathbb{D}}$ of the septum in normal subjects. It may be that 
normal people have some flattening or bowing of the interventricular septum in end-diastole but only in the most cephalic portion. Our data are consistent with the fact that the heart might also rotate about a point on its cephalo-caudal axis in some patients.

Popio et al. (1975) studied angiographic ventricular motion in patients with intra-atrial septal defect by motion analysis of left anterior oblique cineventriculograms along the major axis and along three minor axes. They, therefore, did not study motion with respect to a fixed reference point but rather with respect to a line bisecting the ventricle along its long axis. They found complex multiphasic motion in the midportion of the interventricular septum in midsystole and net contraction of both the septal and posterior walls toward the centre of the ventricle. These findings are compatible with our own, and the multiphasic motions of the interventricular septum in midsystole may be a reflection of the complex motions involved in the reassumption of a rounded minor-axis contour of the ventricle after the interventricular septum has been flattened or bowed into the left ventricular cavity at enddiastole.

We conclude that the abnormal anterior systolic motion of the interventricular septum in patients with intra-atrial septal defect is not found in ventriculographic recordings of interventricular septal motion. In both the patients with intra-atrial septal defect and controls the caudal two-thirds of the interventricular septum, as seen angiographically, move posteriorly in systole. This information favours the hypothesis which holds that the interventricular septum flattens or bows into the left ventricular cavity at end-diastole in conditions of right ventricular overload, and that this anatomical alteration is responsible for the abnormality of interventricular septal motion seen on echocardiography.

\section{References}

Diamond, M. A., Dillon, J. C., Haine, C. L., Chang, S., and Feigenbaum, H. (1971). Echocardiographic features of atrial septal defect. Circulation, 43, 129-135.

Hagan, A. D., Francis, G. S., Sahn, D. J., Karliner, J. S., Friedman, W. F., and O'Rourke, R. A. (1974). Ultrasound evaluation of systolic anterior septal motion in patients with and whthout right ventricular volume overload. Circulation, 50, 248-254.

Hammermeister, K. E., and Warbasse, J. R. (1973). Immediate hemodynamic effects of cardiac angiography in man. American fournal of Cardiology, 31, 307-314.

Kerber, R. E., Dippel, W. F., and Abboud, F. M. (1973). Abnormal motion of the interventricular septum in right ventricular volume overload. Circulation, 48, 86-96.

McCann, W. D., Harbold, N. B., and Giuliani, E. R. (1972). The echocardiogram in right ventricular overload. fournal of the American Medical Association, 221, 1243-1245.

Meyer, R. A., Schwartz, D. C., Benzing, G., III, and Kaplan, S. (1972). Ventricular septum in right ventricular volume overload. An echocardiographic study. American fournal of Cardiology, 30, 349-353.

Pearlman, A. S., Clark, C. E., Henry, W. L., Morganroth, J., Itscoitz, S. B., and Epstein, S. E. (1976). Determinants of ventricular septal motion. Influence of relative right and left ventricular size. Circulation, 54, 83-91.

Popio, K. A., Gorlin, R., Teichholz, L. E., Cohn, P. F., Bechtel, D., and Herman, M. V. (1975). Abnormalities of left ventricular function and geometry in adults with an atrial septal defect. American fournal of Cardiology, 36, 302-308.

Popp, R. L., Wolfe, S. B., Hirata, T., and Feigenbaum, H. (1969). Estimation of right and left ventricular size by ultrasound. A study of echoes from the interventricular septum. American fournal of Cardiology, 24, 523-530.

Weyman, A. D., Wann, S., Feigenbaum, H., and Dillon, J. C. (1976). Mechanism of abnormal septal motion in patients with right ventricular volume overload. A cross-sectional echocardiographic study. Circulation, 54, 179-186.

Requests for reprints to $\mathrm{Dr}$ Thomas M. Mueller, Department of Internal Medicine, University of Iowa Hospitals, Iowa City, Iowa 52242, USA. 